Relations industrielles

Industrial Relations

\title{
Goulet, Mario, Le droit disciplinaire des corporations professionnelles
}

\section{Fernand Morin}

Volume 50, numéro 1, 1995

URI : https://id.erudit.org/iderudit/051006ar

DOI : https://doi.org/10.7202/051006ar

Aller au sommaire du numéro

Éditeur(s)

Département des relations industrielles de l'Université Laval

ISSN

0034-379X (imprimé)

1703-8138 (numérique)

Découvrir la revue

Citer ce compte rendu

Morin, F. (1995). Compte rendu de [Goulet, Mario, Le droit disciplinaire des corporations professionnelles]. Relations industrielles / Industrial Relations, 50(1), 238-239. https://doi.org/10.7202/051006ar

Tous droits réservés (C) Département des relations industrielles de l'Université Laval, 1995
Ce document est protégé par la loi sur le droit d'auteur. L'utilisation des services d'Érudit (y compris la reproduction) est assujettie à sa politique d'utilisation que vous pouvez consulter en ligne.

https://apropos.erudit.org/fr/usagers/politique-dutilisation/ 
bien rare. La conclusion s'ouvre sur ces mutations et incite à une réflexion renouvelée sur le sens que doit maintenant revêtir la liberté du travail (p. 257). Un seul regret du lecteur, compte tenu de l'ampleur de la réflexion de l'auteur sur le travail et le droit: le caractère relativement succinct du passage consacré, dans ce contexte mouvant, au droit du travail. (Quid, par exemple, les vues de A. Gorz, Métamorphoses du travail - Quête de sens, Paris, Galilée, 1988 ?)

D'une façon toute particulière, le lecteur nord-américain pourra se sensibiliser encore davantage au particularisme, à certains égards, du droit du travail français, même si l'ouvrage s'inscrit plus généralement dans l'histoire universelle de cette branche du droit. À la différence d'autres traditions, l'autonomie collective - liberté syndicale, droit de grève, droit à la négociation collective - n'y est pas conçue primordialement en termes "d'organisation communautaire". Elle s'y affirme en tant que liberté publique, au profit de tout salarié. Il en résulte un ordre public social, statut qui loge dans chaque contrat individuel de travail (p. 137). D'où, par exemple, la possibilité pour celui-ci de déroger in mejus à la règle collective (p. 140).

"Petit livre", dixit modestement l'auteur, dont le contenu n'a pu être ici que fort imparfaitement évoqué - chaque page est nourrie d'une réflexion et d'une culture impressionnantes -, mais livre plutôt unique de réflexion essentielle et universelle sur le droit du travail, dans la mesure du moins où il est possible de l'abstraire de la diversité des droits nationaux.

PIERRE VERGE

Université Laval

\section{Le droit disciplinaire des corporations professionnelles}

par Mario Goulet, Cowansville, Éditions Yvon Blais, 1993, 238 p., ISBN 2-89073-888-4.

Compte tenu des critiques fréquentes formulées à l'endroit des corporations professionnelles, l'intérêt d'une étude de l'action disciplinaire au sein de ces organismes publics apparait évident. De plus, qui n'a pas d'idées préconçues au sujet des devoirs et de la responsabilité des professionnels? En raison même de ces préjugés à l'égard des professionnels, ce champ d'étude comporte d'importants et délicats obstacles. Aussi, estce tout à l'honneur de $\mathrm{M}^{\mathrm{e}}$ Mario Goulet d'avoir entrepris pareille aventure.

La faute, élément essentiel, doit d'abord être établie et cette opération implique que l'on circonscrive convenablement les devoirs et obligations des professionnels. Cette opération s'effectue d'abord d'une façon objective puis, en tenant compte de certaines circonstances particulières de chaque affaire. La faute, et ses effets à l'égard des profes- sionnels, des collègues et du public ne peuvent être des données faciles à saisir et à jauger. Ce sont néanmoins les deux pôles de cette courte étude de $M^{e}$ Goulet rédigée initialement en vue de l'obtention d'une maitrise ( $2^{\mathrm{e}}$ cycle) en droit.

La deuxième moitié des deux cent trente-huit pages de l'ouvrage est constituée d'annexes: le Code des professions, une bibliographie, l'index des décisions rapportées, etc. La première partie de cette monographie comprend un grand nombre de citations et de renvois infrapaginales (plus de quatre cents). À ce seul point de vue, il s'agit d'une éclatante démonstration de l'observation de Pascal à l'effet que dans tout texte, il y a souvent plus de bien des autres que du sien. Pareille facture présentée pour les fins d'un deuxième cycle d'études universitaires demeure 
fort compréhensible. Mais, il devrait peut être en être autrement lorsqu'on offre au public semblable ouvrage sous un titre quasi générique: "Le droit disciplinaire des corporations professionnelles "!

Le chapitre préliminaire traite du caractère autonome du droit disciplinaire (p. 9 à 34). L'auteur déclare qu'il y élabore une "théorie de l'autonomie du droit disciplinaire" (p. 9). Au-delà des sous-titres et de quelques assertions, nous n'y avons guère trouvé pareille élaboration, mais seulement une courte démonstration du particularisme de ce volet spécifique du droit. Distinct du droit pénal, exclu du droit privé, ce droit disciplinaire est certes une composante du droit administratif et soumis au respect des chartes des droits et libertés. Il est, en quelque sorte, l'armature juridique au soutien de la déontologie des professionnels. Compte tenu de sa spécificité, ce processus de contrôle par ses pairs n'écarte pas l'application parallèle de la justice pénale ou civile: "Un professionnel peut être poursuivi pour le même acte, ou la même omission, à la fois au pénal, au civil et devant une instance disciplinaire, car une faute disciplinaire ne constitue pas nécessairement une infraction pénale et n'entraîne pas nécessairement une responsabilité civile. L'inverse est également vrai " ( $p$. 24).

La première partie de l'ouvrage ( $p$. 35 à 82) porte sur la faute disciplinaire que l'on définit comme un "manquement volontaire ou d'une certaine gravité à un devoir professionnel " (p. 39). À notre avis, le caractère expressément volontaire d'un acte fautif professionnel aggrave certes l'acte reprochê mais celuici est néanmoins un manquement! On souligne cependant que le manquement reproché doit pouvoir être normalement et raisonnablement déduit du code de déontologie de la profession visée: "Le texte devrait ētre compréhensible aux yeux du professionnel et correspondre à un devoir d'ordre général, tel qu'édicté par le pouvoir habilitant " (p. 41). L'auteur explique le caractère juridique de la faute professionnelle et qu'il distingue de la faute pénale (p. 46). Ce manquement professionnel est rattaché à la personne même de l'auteur sans égard au lieu où il est réalisé, ni au contexte collectif qui l'explique (exemple : la grève) (p. 48) et ni même au fait qu'il entraîne ou non des dommages à d'autres personnes (p. 49). Le chapitre II (p. 61 à 82 ), serait une classification des fautes disciplinaires. Sous ce titre, on y retrouve davantage une synthèse de la jurisprudence de différents comités de discipline et du Tribunal des professions. Ces manquements sont sériés en trois catégories : compétence, intégrité et dignité.

La deuxième partie du livre (p. 83 à 115) porte sur la sanction disciplinaire. L'auteur démontre que l'approche alors retenue par les comités de discipline et le Tribunal des professions serait de nature pénale et regrette une telle conception qui occulte l'effet correctif de la sanction: "Le but du contrôle disciplinaire n'est pas la punition mais bien la protection du public " (p. 84). Dans sa conclusion, l'auteur reprend le thème de l'autonomie du droit disciplinaire sans davantage justifier ni expliciter le sens et la portée de cette autonomie.

FERNAND MORIN Université Laval

\section{Women, Work, and Coping}

edited by Bonita C. LONG and Sharon E. KAHN, Toronto, McGill-Queen's

University Press, 1993, 332 p., ISBN 0-7735-1128-8 (cloth),

ISBN 0-7735-1129-6 (paper).

Parmi les femmes qui sont confrontées au stress dans leur emploi, certai- nes en retirent une plus grande satisfaction que d'autres qui ne travaillent pas 